\title{
Sex specific expression and distribution of small RNAs in papaya
}

Rishi Aryal', Guru Jagadeeswaran ${ }^{2}$, Yun Zheng ${ }^{3}$, Qingyi Yu ${ }^{4,5}$, Ramanjulu Sunkar ${ }^{2^{*}}$ and Ray Ming ${ }^{1,5^{*}}$

\begin{abstract}
Background: Regulatory function of small non-coding RNAs (sRNA) in response to environmental and developmental cues has been established. Additionally, sRNA, also plays an important role in maintaining the heterochromatin and centromere structures of the chromosome. Papaya, a trioecious species with recently evolved sex chromosomes, has emerged as an excellent model system to study sex determination and sex chromosome evolution in plants. However, role of small RNA in papaya sex determination is yet to be explored.

Results: We analyzed the high throughput sRNAs reads in the Illumina libraries prepared from male, female, and hermaphrodite flowers of papaya. Using the sRNA reads, we identified 29 miRNAs that were not previously reported from papaya. Including this and two previous studies, a total of 90 miRNAs has been identified in papaya. We analyzed the expression of these miRNAs in each sex types. A total of 65 miRNAs, including 31 conserved and 34 novel mirNA, were detected in at least one library. Fourteen of the 65 miRNAs were differentially expressed among different sex types. Most of the miRNA expressed higher in male flowers were related to the auxin signaling pathways, whereas the miRNAs expressed higher in female flowers were the potential regulators of the apical meristem identity genes. Aligning the sRNA reads identified the sRNA hotspots adjacent to the gaps of the $X$ and $Y$ chromosomes. The $X$ and $Y$ chromosomes sRNA hotspots has a 7.8 and 4.4 folds higher expression of sRNA, respectively, relative to the chromosome wide average. Approximately $75 \%$ of the reads aligned to the $X$ chromosome hotspot was identical to that of the $Y$ chromosome hotspot.

Conclusion: By analyzing the large-scale sRNA sequences from three sex types, we identified the sRNA hotspots flanking the gaps of papaya $X, Y$, and $Y^{h}$ chromosome. The sRNAs expression patterns in these regions were reminiscent of the pericentromeric region indicating that the only remaining gap in each of these chromosomes is likely the centromere. We also identified 14 differentially expressed miRNAs in male, female and hermaphrodite flowers of papaya. Our results provide valuable information toward understanding the papaya sex determination.
\end{abstract}

Keywords: Carica papaya, Centromere, miRNA, siRNA, Sex chromosome, Sex determination

\section{Background}

Micro RNA (miRNA) and small interfering RNA (siRNA) are two major classes of endogenous regulatory RNAs (sRNA) found in higher plants. These sRNAs are processed from RNA duplexes by a dicer family protein, which produces approximately 21-24nt final products. Many miRNAs regulate various developmental processes by sequence directed silencing of the mRNA at a posttranscriptional level

\footnotetext{
* Correspondence: ramanjulu.sunkar@okstate.edu; rming@life.illinois.edu ${ }^{2}$ Department of Biochemistry and Molecular Biology, Oklahoma State University, Stillwater, OK 74078, USA

'Department of Plant Biology, University of Illinois at Urbana-Champaign, Urbana, IL 61801, USA

Full list of author information is available at the end of the article
}

[1-5]. The siRNAs regulate the genome function both at transcriptional and posttranscriptional levels through RNA guided DNA methylation and RNA guided RNA silencing, respectively [6-9].

Spatial and temporal pattern of organ formation in plants are regulated at various levels by sRNA mediated gene silencing. The development of the male and female gametophyte is regulated by the combinatorial action of various sRNAs [10,11]. In Arabidopsis, early embryo patterning and transition from juvenile to adult plant is regulated by miR156 [12-14]. In rice, Arabidopsis, and maize, mir166 regulates the adaxial/abaxial patterning of the leaves [15]. Micro-RNAs and other sRNAs are important regulators of flower development and floral 
organ identity in many plant species [13,16-21]. Further analysis of sRNA transcriptome in various organs, tissues, and developmental phases will provide a better understanding on their function in plant development and organogenesis.

Previous reports suggest that miRNAs play a role in sex differentiation in plants [21-23]. Differential expression of miRNAs that regulates the development of carpels and stamens can ultimately lead to the development of unisexual flowers and to the sexual differentiation in plants. In maize, miR172 maintains the unisexual nature of the tassel by suppressing carpel development genes Ts6 [23]. In Petunia hybrida and Antirrhinum majus, the miR169 family genes, miRBL and miRFIS, restrict the expression of $C$ class homeotic genes at the center of the flower. Plants defective in these miRNA genes produce the female flowers on genetically hermaphroditic individuals [18]. Small RNAs are also important in maintaining the integrity of sex chromosomes by methylating the non-recombining regions during meiotic prophase [24].

Papaya (Carica papaya) is a model species to study the plant sex determination. It is a trioecious species with three sex types, hermaphrodite, male, and female. It belongs to the family Caricaceae, which comprises 35 species in six genera including one monoecious, 32 dioecious, and two trioecious species. Papaya diverged from its closest monoecious relative (Vasconcellea monoica) about 27.5 million years ago [25]. Sex expression in papaya is controlled by a pair of recently evolved sex chromosomes. The male and hermaphrodite characteristics are determined by two slightly different Y-chromosomes, $\mathrm{Y}$ and $\mathrm{Y}^{\mathrm{h}}$, respectively $[26,27]$. The genotype XX determines female, XY determines male, and $X Y^{\mathrm{h}}$ determines hermaphrodite. All combinations of $Y$ and $Y^{\mathrm{h}}$ are embryonic lethal, indicating that the $\mathrm{Y}$ and $\mathrm{Y}^{\mathrm{h}}$ chromosomes have lost some genes necessary for embryo development [26]. The $\mathrm{Y}$ and $\mathrm{Y}^{\mathrm{h}}$ chromosomes show $98.9 \%$ sequence similarity on average, differing mainly in intergenic and repetitive regions [28]. The male specific region of Y chromosome (MSY) and hermaphrodite specific region of $\mathrm{Y}^{\mathrm{h}}$ (HSY) are highly methylated and heterochromatized compared to the corresponding region of $\mathrm{X}$ chromosome [29]. Because sRNA is involved in establishing heterochromatic structures, analyzing the sRNA transcripts from the papaya sex chromosome will help to understand the epigenetic aspects of the recently evolved sex chromosomes.

Sequencing the HSY and its corresponding region in the $\mathrm{X}$ chromosome have produced 8.1 and $3.4 \mathrm{Mb}$ pseudomolecules for the sex determining regions of the respective chromosomes [30,31]. Sequence alignment between HSY and corresponding region of the $\mathrm{X}$ chromosome revealed two large-scale inversions and several intrachromosomal rearrangements were identified on the HSY, however precise location of these inversions are still unknown due to an unfilled gap on the physical map. In the X chromosome, the repeat density gradually increases towards the gap, suggesting that the gap is likely the centromere [31]. Using the fluorescent in situ hybridization technique, the centromere of the Y chromosomes was identified near the knob 4 [29]. The knob 4 region is now completely sequenced, however, analysis of repeat content in the region did not identify centromeric satellite elements in this region [30]. These conflicting observations call for the search of centromere on the $\mathrm{Y}$ chromosomes. Identification of centromere will provide valuable information regarding the location of inversion and direction of sequence expansion at the early stage of papaya sex chromosome evolution.

We previously analyzed the genome-wide sRNA profile in papaya using the high throughput Illumina sRNA libraries prepared from flowers and leaves including Papaya Ringspot Virus (PRSV) infected leaves [32]. Evidences from many plant species show that sex determination in plants are also regulated by epigenetic mechanisms including that of small noncoding RNAs [33]. In this study, we analyzed the sRNAs libraries prepared from male, female, and hermaphrodite flowers of papaya. We used the high throughput sRNA sequences to identify the miRNAs in different sex types. Expression of miRNAs in the flowers of three papaya sex types is analyzed. Aligning the sRNA reads identified the sRNA hotspots on papaya sex chromosomes similar to that of pericentromeric region. We discuss the early events in papaya sex chromosome evolution in light of the identified centromeric location.

\section{Results}

\section{Sequencing sRNAs from papaya flowers}

Small RNA libraries were constructed from male, female, and hermaphrodite flowers and sequenced using the Illumina Genome Analyzer II. After adapter trimming and removing the low quality reads, a total of 1.6 million reads from male, 3.6 million reads from female, and 1.8 million reads from hermaphrodite flowers were obtained for further analysis (Table S1). The sRNA libraries were mainly represented by $21 \mathrm{nt}$ and $24 \mathrm{nt}$ species (Figure 1). The 21 and $24 \mathrm{nt}$ sRNA constituted $20 \%$ and $36 \%$ of the unique reads in male library, $16 \%$ and $46 \%$ of the unique reads in female library, and $18 \%$ and $37 \%$ of the unique reads in hermaphrodite library, respectively.

We previously reported a higher accumulation of purinerich strands (sequences with more purine residue than pyrimidine residue) than the pyrimidine-rich strands (sequences with more pyrimidine residue than purine residue) in sRNA libraries from various plant species [32]. To further confirm this phenomenon, we analyzed the purine-rich and pyrimidine-rich sequences in the three libraries. In congruence with the previous observation, all three libraries were overrepresented by purine- 


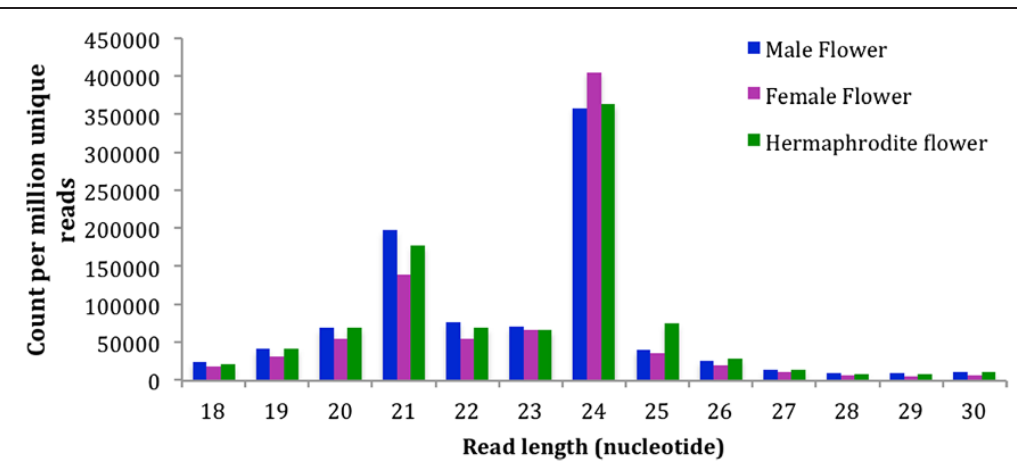

Figure 1 Size distribution of sRNA reads from three libraries. Unique sequences were obtained from each library after adapter trimming and removing other known classes of contaminant RNAs.

rich strands (Additional file 1: Figure S1). Purine-rich sequences constituted $62 \%, 65 \%$, and $65 \%$ of the total reads in male, female and hermaphrodite libraries, respectively, whereas pyrimidine-rich sequences constituted 30\%, 28\% and $25 \%$ in the respective libraries. Sequences with equal number of purine and pyrimidine residues were found $8 \%$ in the male library, $7 \%$ in female the library, and $10 \%$ in the hermaphrodite library. To see whether the purinepyrimidine bias is localized on a particular region or is spread across the sRNA sequences, we analyzed the frequency of nucleotides in each position of 21nt and 24nt sequences (Additional file 1: Figure S2). Adenine was the most frequent nucleotide throughout the $24 \mathrm{nt}$ sequence, whereas $21 \mathrm{nt}$ sequences were overrepresented by guanine nucleotides. Uracil and adenine was highly conserved at the 5 ' end of $21 \mathrm{nt}$ and $24 \mathrm{nt}$ sequences, respectively.

\section{Identification of miRNAs from the sRNA libraries}

Since the expression of miRNA is highly influenced by various environmental and developmental factors, an extensive search in various tissues, developmental stage, genotype etc. is required to get a complete profile of miRNAs in a species. We utilized the high throughput sRNA reads obtained from the flowers of different sex types to search for the miRNAs previously not identified in papaya. Conserved miRNAs were identified based on the homology with previously identified miRNAs from other species. Novel miRNAs were identified if the effector strand (miRNA) and complementary strand (miRNA*) sequences were present in the libraries, and meet the miRNA annotation criteria suggested by Meyers et al. [34]. Small RNA reads analysis of these three different libraries led to the identification of 29 miRNAs that includes 10 conserved miRNA homologs and 19 novel miRNAs (Table 1, Additional file 1: Figure S3), beside the 61 miRNAS reported previously $[32,35]$. Taken together, two previous studies and this study, a total of 90 miRNAs are identified in papaya, of which 34 belong to conserved miRNAs and the remaining 56 are novel miRNAs.

\section{MiRNA abundance in papaya flowers}

We analyzed the abundance of identified miRNAs in the sRNA libraries. A total of 65 (31 conserved and 34 novel) miRNAs were detected in at least one of the three sRNA libraries (Table 2). Of the 65 miRNAs, 31 were expressed more than 100 copies per million reads in at least one of the three libraries. We included only those 31 miRNAs for further expression analysis. None of the 31 miRNAs was specific to one sex type, however, at least two-fold difference in abundance among the three sex types was detected for 14 miRNAs (Figure 2). Of the 14 differentially expressed miRNAs, six miRNAs (miR160, miR167a, miR167b, miR393, miR169, miR_novel_10) were more abundant in male flowers, compared to female and hermaphrodite flowers. Five of the six miRNAs (miR160, miR167a, miR167b, miR169 and miR393) have been shown to regulate auxin-signaling pathway [36-38]. MiR169 was expressed more than 5 folds higher in male and $\sim 2$ folds higher in hermaphrodite flowers compared to female flowers. Four miRNAs (miR156a, miR156b, miR168 and miR_novel_39) showed higher abundance in male and hermaphrodite flowers than in female flowers. More contrasting difference was observed for miR156 family (miR156a, and miR156b) with $\sim 5$ folds higher abundance in male and hermaphrodite flowers. MiR164 was expressed higher in female flowers relative to the other two samples. Two miRNAs, miR159 and miR166, showed higher overall abundance and higher abundance in female flowers compared to male and hermaphrodite flowers, but the difference in abundance level was less than 2 folds. Two miRNAs, miR171 and miR394, showed higher abundance in male and female flowers compared to the hermaphrodite flowers. A novel miRNA, miR_novel_06, was expressed higher in hermaphrodite sample than in male and female samples.

In general, conserved miRNAs were expressed higher in all sex types than the novel miRNAs. Of the 34 novel miRNAs, only 7 were expressed more than 100 copies, whereas 24 of the 31 conserved miRNAs were expressed 
Table 1 List of papaya miRNAs identified in this study

\begin{tabular}{|c|c|c|c|c|}
\hline MiRNA family & miR_sequence & Strand & Chromosome & miR location \\
\hline CpmiR397 & UCAUUGAGUGCAGCGUUGAUGU & - & Supercontig_27 & $1548952 . .1548973$ \\
\hline CpmiR398a & UGUGUUCUCAGGUCACCCCUU & + & Supercontig_1 & $1672760 . .1672780$ \\
\hline CpmiR398b & UGUGUUCUCAGGUCGCCCCUG & + & Supercontig_34 & $1614486 . .1614506$ \\
\hline CpmiR399a & UGCCAAAGGAGAUUUGCCCGG & + & Supercontig_7 & $849330 . .849350$ \\
\hline CpmiR399b & UGCCAAAGGAGAUUUGCCCGG & - & Supercontig_7 & $855497 . .855517$ \\
\hline CpmiR399c & UGCCAAAGGAGAGUUGCCCUG & + & Supercontig_4 & $3751380 . .3751400$ \\
\hline CpmiR403 & UUAGAUUCACGCACAAACUCG & $\mathrm{n} / \mathrm{a}$ & $\mathrm{n} / \mathrm{a}$ & $\mathrm{n} / \mathrm{a}$ \\
\hline CpmiR894 & CGUUUCACGUCGGGUUCACC & $\mathrm{n} / \mathrm{a}$ & $\mathrm{n} / \mathrm{a}$ & $\mathrm{n} / \mathrm{a}$ \\
\hline CpmiR2111 & UAAUCUGCAUCCUGAGGUUUA & + & Supercontig_171 & $301214 . .301234$ \\
\hline CpmiR2910 & UAGUUGGUGGAGCGAUUUGUC & + & Supercontig_0 & $2421938 . .2421958$ \\
\hline CpmiR-novel_36 & UGGUCAACUUCACUAAUGCUUU & + & Supercontig_140 & $285663 . .285642$ \\
\hline CpmiR-novel_37 & AGAUAAAUCAGAGGAUCUAACC & + & Supercontig_27 & $1714759 . .1714738$ \\
\hline CpmiR-novel_38 & UUGCCAUUGCUGUCAUCAUUG & - & Supercontig_26 & $1264911 \ldots 1264891$ \\
\hline CpmiR-novel_39 & UUCGCCAGCCAUUCACAAAAU & - & Supercontig_67 & $294009 . .293989$ \\
\hline CpmiR-novel_40 & UGCAGUAUCUGUAGCAUCAGC & + & Supercontig_1867 & 3234.3214 \\
\hline CpmiR-novel_41 & UUAUGCAGAUACCCGGAGGAG & + & Supercontig_2379 & $8824 . .8804$ \\
\hline CpmiR-novel_42 & CAGAGGAGGAGAUGAAGAGGGA & - & Supercontig_609 & $25591 . .25570$ \\
\hline CpmiR-novel_43 & UAAGACAAAGCCUACAACAAC & - & Supercontig_92 & $867729 . .867709$ \\
\hline CpmiR-novel_44 & UGGGAUCCAGUGCAUUAGUGC & + & Supercontig_241 & $21475 . .21455$ \\
\hline CpmiR-novel_45 & AUUGGAGGACUUUGGGGGAGC & - & Supercontig_282 & 4156.4136 \\
\hline CpmiR-novel_46 & UCUUGCAAGCUGCUUAGAUCA & - & Supercontig_130 & $392538 . .392518$ \\
\hline CpmiR-novel_47 & UUUCUACCCACCUUUACCUCCGUG & - & Supercontig_425 & 30640.30617 \\
\hline CpmiR-novel_48 & CAGAAGUAAAGGUUGGUAGAAAA & - & Supercontig_77 & $529407 . .529385$ \\
\hline CpmiR-novel_49 & UUUUGGGACACGUGCAGGUAC & + & Supercontig_26 & $75358 . .75338$ \\
\hline CpmiR-novel_50 & CUGCGUAUAAAUUUUGCUCCG & + & Supercontig_271 & 79608..79588 \\
\hline CpmiR-novel_51 & UUUCCAAAUUCUCUCGUACCGA & + & Supercontig_65 & $875942 . .875921$ \\
\hline CpmiR-novel_52 & AGGCGCACUGUGAAUCGUAUUCGG & + & Supercontig_33 & $742481 . .742458$ \\
\hline CpmiR-novel_53 & AUCUGGGCCGUCCGUGCGCAC & - & Supercontig_74 & $405778 . .405758$ \\
\hline CpmiR-novel_54 & UACCGGACGAAGUAUCGAGACGAU & - & Supercontig_246 & $189341 . .189318$ \\
\hline
\end{tabular}

The novel miRNAs are named contiguous from our previous report [32].

more than 100 copies. MiR166 showed highest overall expression followed by miR156 and miR159.

\section{Small RNA landscape on papaya sex chromosomes}

To analyze the sRNA transcriptome of the sex chromosomes, we mapped the sRNA reads from each library to the respective pseudomolecules [30]. The adapter trimmed non-redundant (unique) reads were aligned to the male specific region of Y chromosome (MSY), HSY, and the corresponding region on $\mathrm{X}$ chromosome. Only the reads with $100 \%$ sequence alignment were taken for the downstream analysis. The number of reads mapped to the MSY was higher than that of HSY. Approximately $3.5 \%$ of the unique reads from each library were aligned to the HSY, $5.5 \%$ to the MSY, and $4 \%$ to the X chromosome (Additional file 1: Table S1).
The physical map of MSY and HSY contains a gap at the border ' $\mathrm{A}$ ' region (border ' $\mathrm{A}$ ' represents the left border and border ' $\mathrm{B}$ ' represents the right border in each chromosome) [30,39]. Similarly, the corresponding region of the $\mathrm{X}$ chromosome contains a large gap in the middle of the physical map. We observed a 7.8 and 4.4 folds higher expression of sRNAs adjacent to the gaps on each of the $\mathrm{Y}$ and $\mathrm{X}$ chromosomes compared to the chromosome wide average, respectively (Figure 3). Approximately $19 \%$ of the unique reads were aligned to the first $350 \mathrm{~kb}$ of the MSY and HSY pseudomolecules (4.3\% of the pseudomolecules). Similarly, approximately $14 \%$ of unique reads aligned to $\mathrm{X}$ chromosome were mapped to the $60 \mathrm{~kb}$ region spanning the gap $(30 \mathrm{~kb}$ on each side; a total of $1.8 \%$ of the pseudomolecule). Elevated expression of sRNA near the gaps on the papaya sex 
Table 2 Normalized expression of miRNA in flowers of different sex types

\begin{tabular}{|c|c|c|c|}
\hline \multirow[t]{2}{*}{ MiRNA family } & \multicolumn{3}{|c|}{ Expression (counts per million reads) } \\
\hline & Male & Female & Hermaphrodite \\
\hline CpmiR156/157a & 36079 & 5385 & 30491 \\
\hline CpmiR156/157b & 36378 & 7122 & 30392 \\
\hline CpmiR159 & 8069 & 13077 & 8734 \\
\hline CpmiR160 & 3291 & 1647 & 854 \\
\hline CpmiR162 & 214 & 286 & 193 \\
\hline CpmiR164 & 311 & 891 & 252 \\
\hline CpmiR165/166a & 48299 & 74721 & 30625 \\
\hline CpmiR165/166b & 26839 & 38348 & 31875 \\
\hline CpmiR167a & 6320 & 2433 & 2449 \\
\hline CpmiR167b & 6334 & 1824 & 2585 \\
\hline CpmiR168 & 5124 & 2369 & 8676 \\
\hline CpmiR169 & 2738 & 576 & 1060 \\
\hline CpmiR171 & 1515 & 1079 & 506 \\
\hline CpmiR172 & 6342 & 4544 & 5014 \\
\hline CpmiR390 & 2095 & 1328 & 2109 \\
\hline CpmiR393 & 900 & 53 & 61 \\
\hline CpmiR394 & 483 & 667 & 189 \\
\hline CpmiR395 & 17 & 26 & 34 \\
\hline CpmiR396 & 3381 & 2149 & 2402 \\
\hline CpmiR397 & 5 & 19 & 31 \\
\hline CpmiR398a & 13 & 6 & 14 \\
\hline CpmiR398b & 9 & 17 & 72 \\
\hline CpmiR399a & 6 & 1 & 1 \\
\hline CpmiR399b & 2 & 1 & 2 \\
\hline CpmiR399c & 2 & 1 & 2 \\
\hline CpmiR403 & 121 & 68 & 96 \\
\hline CpmiR408 & 145 & 82 & 93 \\
\hline CpmiR535 & 2852 & 4756 & 4368 \\
\hline CpmiR894 & 308 & 272 & 250 \\
\hline CpmiR2111 & 25 & 243 & 277 \\
\hline CpmiR2910 & 161 & 195 & 340 \\
\hline CpmiR-novel_01 & 12 & 14 & 3 \\
\hline CpmiR-novel_02 & 84 & 24 & 26 \\
\hline CpmiR-novel_03 & 3 & 17 & 8 \\
\hline CpmiR-novel_04 & 19 & 17 & 14 \\
\hline CpmiR-novel_05 & 66 & 11 & 18 \\
\hline CpmiR-novel_06 & 954 & 621 & 1613 \\
\hline CpmiR-novel_09 & 8 & 19 & 11 \\
\hline CpmiR-novel_10 & 106 & 6 & 17 \\
\hline CpmiR-novel_11 & 93 & 94 & 92 \\
\hline CpmiR-novel_12 & 0 & 0 & 5 \\
\hline CpmiR-novel_17 & 1 & 2 & 2 \\
\hline
\end{tabular}

Table 2 Normalized expression of miRNA in flowers of different sex types (Continued)

\begin{tabular}{|c|c|c|c|}
\hline CpmiR-novel_25 & 1 & 2 & 2 \\
\hline CpmiR-novel_26 & 0 & 3 & 1 \\
\hline CpmiR-novel_28 & 1 & 1 & 1 \\
\hline CpmiR-novel_33 & 13 & 2 & 4 \\
\hline CpmiR-novel_36 & 9 & 3 & 1 \\
\hline CpmiR-novel_37 & 126 & 230 & 239 \\
\hline CpmiR-novel_38 & 131 & 67 & 69 \\
\hline CpmiR-novel_39 & 1390 & 741 & 1806 \\
\hline CpmiR-novel_40 & 128 & 7 & 15 \\
\hline CpmiR-novel_41 & 4 & 7 & 18 \\
\hline CpmiR-novel_42 & 19 & 5 & 5 \\
\hline CpmiR-novel_43 & 8 & 3 & 3 \\
\hline CpmiR-novel_44 & 7 & 16 & 29 \\
\hline CpmiR-novel_45 & 28 & 28 & 34 \\
\hline CpmiR-novel_46 & 259 & 268 & 131 \\
\hline CpmiR-novel_47 & 2 & 4 & 4 \\
\hline CpmiR-novel_48 & 20 & 16 & 15 \\
\hline CpmiR-novel_49 & 12 & 17 & 11 \\
\hline CpmiR-novel_50 & 15 & 3 & 5 \\
\hline CpmiR-novel_51 & 3 & 2 & 2 \\
\hline CpmiR-novel_52 & 161 & 141 & 105 \\
\hline CpmiR-novel_53 & 5 & 1 & 1 \\
\hline CpmiR-novel_54 & 3 & 5 & 2 \\
\hline
\end{tabular}

chromosome prompted a new hypothesis that these regions may represent the pericentromeric region of the chromosome. Furthermore, the pericentromeric sRNAs are highly conserved among the chromosomes in the yeast species, Schizosaccharomyces pombe [40]. To reaffirm whether the observed sRNA hotspots on the sex chromosomes are actually the pericentromeric region, we analyzed the conservation of the sRNAs between the putative pericentromeric regions of the $\mathrm{X}$ and $\mathrm{Y}$ chromosomes. Approximately $63 \%$ of the sRNAs aligned to the pericentromeric region of the $\mathrm{Y}$ chromosome were also aligned to the pericentromeric region of the $\mathrm{X}$ chromosome. Similarly, approximately $76 \%$ of the sRNAs aligned to pericentromeric region of the $\mathrm{X}$ chromosome also aligned to the pericentromeric region of the $\mathrm{Y}$ chromosome.

The papaya $\mathrm{Y}$ chromosomes differ from the $\mathrm{X}$ chromosome by two large scale inversions [30]. However, the precise location of inversion remained unclear due to the gap on both $\mathrm{X}$ and $\mathrm{Y}$ chromosomes. If the centromere of the $\mathrm{X}$ and $\mathrm{Y}$ chromosomes is at the respective gap of the physical map, the two centromeres are located approximately 1.6 Mb apart from each other. Together, these data indicate that the first inversion on the $\mathrm{Y}$ 


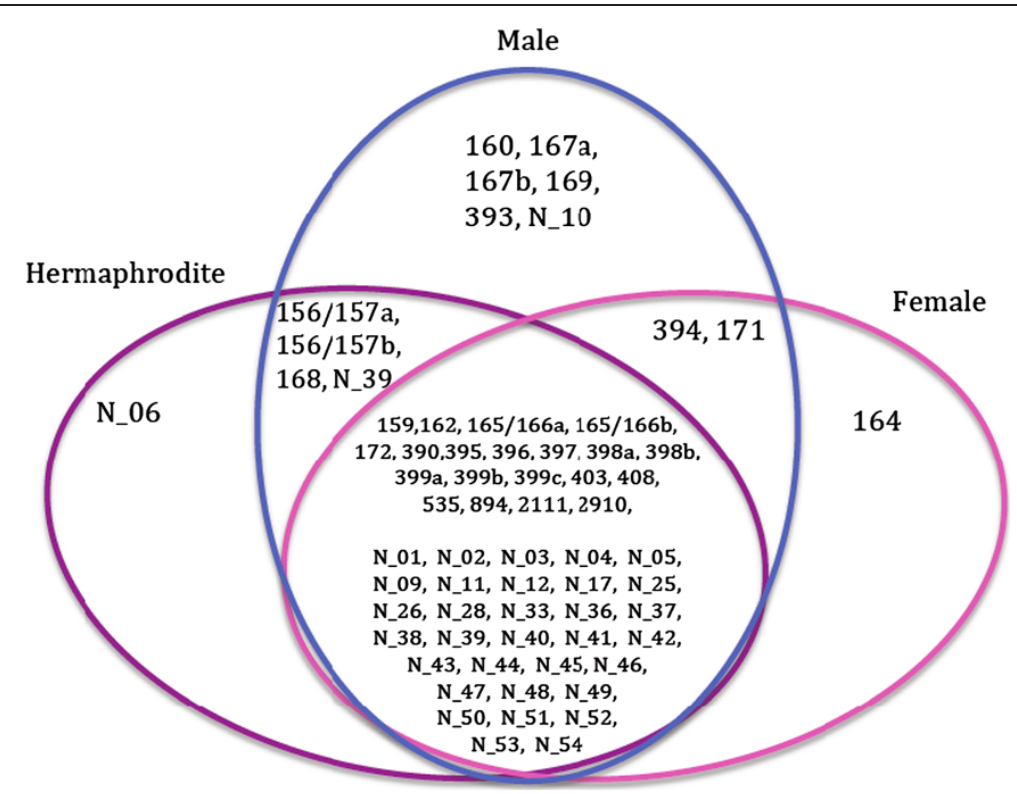

Figure 2 Differentially expressed of miRNAs in the flowers of three papaya sex types. The miRNA presented in each sex type(s) is expressed at least 2-fold higher in that sex type(s). The miRNA listed at the center has no differential expression among the sex types. N_denotes the novel miRNA.

chromosome occurred at pericentric region spanning the centromere (Figure 4). To test this hypothesis, we searched the protein coding genes adjacent to the gaps on $\mathrm{Y}$ and $\mathrm{X}$ chromosomes. One X specific gene (CpX24) was found $65.5 \mathrm{~kb}$ away from the gap towards the boarder 'B'. Search for the homologous region of this gene on $\mathrm{Y}$ chromosome did not yield any result, indicating that the corresponding Y copy of this gene may have moved with the first inversion. There was no protein coding genes located in the first $478 \mathrm{~kb}$ region adjacent to the gap on $\mathrm{Y}$ chromosome.

\section{Discussion}

The papaya MSY and HSY have been mapped near the centromere of both $\mathrm{Y}$ and $\mathrm{Y}^{\mathrm{h}}$ chromosomes [41-43]. A physical map of the MSY and HSY has been generated, but a large unfilled gap remains at the 'border A' region. Similarly, a gap remains in the middle of the $\mathrm{X}$ chromosome

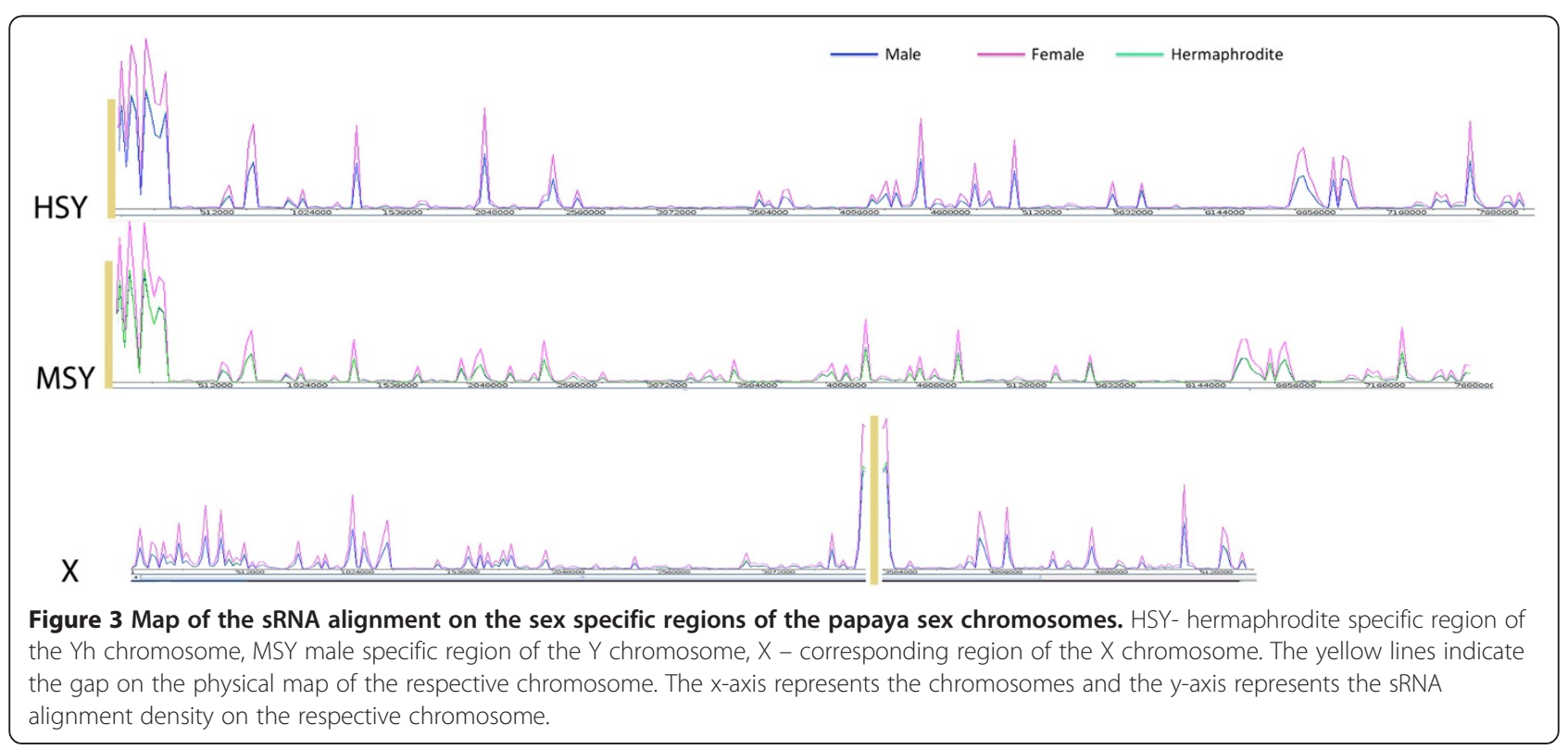




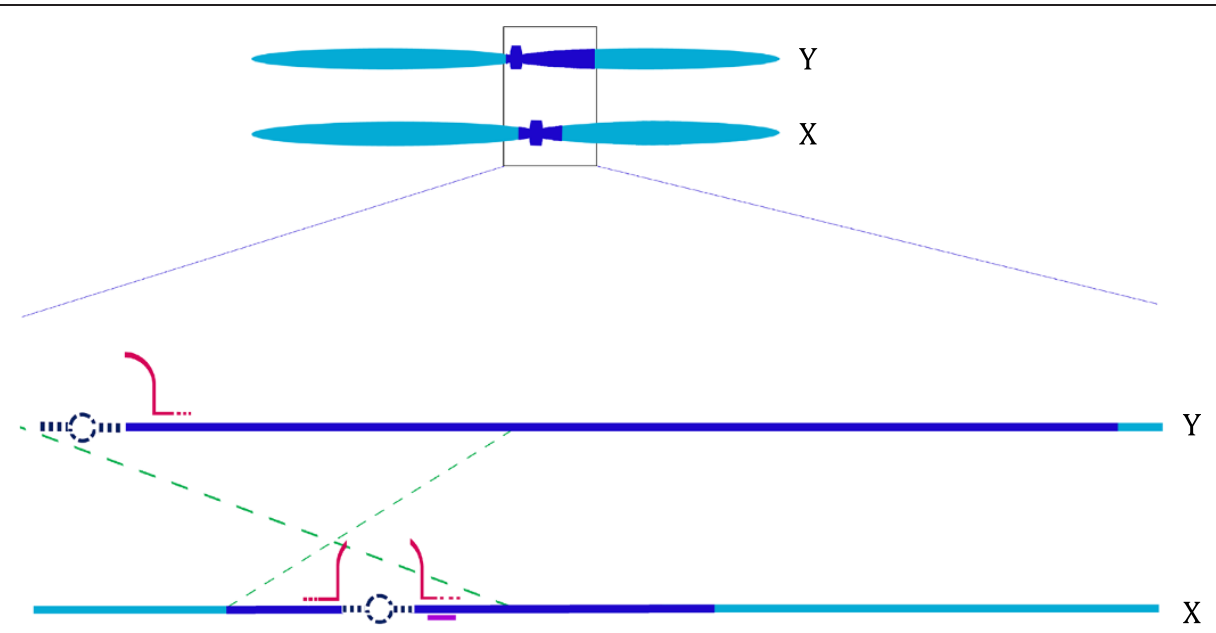

Figure 4 Diagrammatic representation of papaya sex chromosomes showing putative centromeres and inversion region. The sex specific regions are shown on dark blue and pseudo-autosomal regions are shown on light blue. Bottom panel shows the zoomed view of sex determining region. The blue dotted line represents the gap on physical map with the putative centromere represented by dotted circle. The red curves indicate the higher sRNA expressing loci adjacent to the gaps; the purple bar below $X$ chromosome represents an $X$ specific genes. The green dotted lines indicate approximate position for the inversion I [30] on the $Y$ chromosome relative to the $X$ chromosome.

physical map [30,39]. Analysis of repetitive sequences provided strong evidence that the centromere is located in the middle of the $\mathrm{X}$ chromosome where the gap remains on the physical map [31]. We observed a sharp increase in sRNA expression at both edges of the gap in the X chromosome, which further strengthened the notion that the gap is the centromere. For the $\mathrm{Y}$ chromosomes, it was suggested that the centromere might be at either side of Knob 4 in the HSY based on fluorescent in situ hybridization of Knob-specific BACs on anaphase chromosomes [29]. If this were the case, we would have mapped and sequenced the centromere as there is no gap in that region [30,39], but there is no long tandem repeats nor the rise and fall of repetitive sequences, typical features of centromeres. Our evidence of sharp increase of sRNA at the edge of gap in the HSY and MSY strongly supports that the only remaining gap in the physical map is likely the centromere of the $Y$ chromosomes (Figure 3). Identification of the Y chromosome centromere is a major advancement on papaya sex chromosome research, and set the stage for further characterization and eventual sequencing of the $Y$ centromere. This finding also indicated that the first inversion that triggered sex chromosome evolution is pericentric.

The gap in HSY and MSY include a large genomic region corresponding to the Knob 1 that shared between $\mathrm{X}$ and $\mathrm{Y}$ chromosomes, and chromosome walking on the HSY kept landing on the $\mathrm{X}$ chromosome counterpart, which was mapped and sequenced. The next question would be how extensive of the pericentric region on the unmapped side of the gap in HSY and MSY. The question can be addressed by examine the $\mathrm{X}$ specific genes between the two evolutionary strata in the $\mathrm{X}$ chromosome. Analysis of $\mathrm{X}$ specific genes between genes corresponding to the edges of inversions 1 and 2 revealed only one $\mathrm{X}$ specific gene, suggesting that the unmapped side of gap in the HSY and MSY involved few genes in the Inversion 1, and consider faster rate of gene loss in the HSY [30], it could be only one (paired with the one $\mathrm{X}$ specific gene between the two strata) or none gene on the unmapped side of the centromere in inversion 1.

Higher expression sRNAs at the pericentromeric region of the chromosome has been observed in other plant species [44-46]. The sRNA sequences aligned to the pericentromeric regions were highly conserved between $\mathrm{X}$ and $\mathrm{Y}$ chromosomes with up to $76 \%$ of sequence mapped to these regions being common in both, providing further evidence for the location of the centromere in the respective gaps. High conservation among the sRNA sequences expressed from the pericentromeric regions of different chromosomes was also observed in the yeast centromeres [40]. The centromeric sequences of the chromosomes remain elusive in the assemblies of many genomes due to their highly repetitive nature [47]. To date, complete sequence for the centromeric region is available only for Chromosome 8 of rice that was recently evolved [48]. The fact that only one gap each remaining in the papaya X, HSY and MSY physical maps validates the high quality of these physical maps and also indicated that these centromeres are likely the original centromere of the autosomes that these sex chromosomes evolved from. The alignment of candidate centromere position between papaya $\mathrm{X}$ chromosome and the orthologous autosome of $V$. monoica is in line with this conclusion. The inversion and degeneration of the $\mathrm{Y}$ chromosome did not destroy or caused turnover of the Y chromosome centromere. 
Premature separation of the papaya sex chromosomes compared to the autosome has been detected in meiotic anaphase [49]. This premature separation is likely the consequence of suppressed recombination in the sex specific region and improper alignment of the centromere due to their shifted position relative to each other caused by the pericentric inversion.

A total of 14 miRNAs were differentially expressed among male, female, and hermaphrodite flowers, indicating their potential function in papaya sex determination (Table 2, Figure 2). All conserved miRNAs that are expressed highest in male flowers (miR160, miR167a, miR167b, miR169, and miR393) regulates the genes in auxin signaling pathway [36-38,50]. Experimental evidences indicate that auxin plays a central role in carpel development (see [51] for more review). Additionally, miR169 regulates floral development in Nocotiana benthamiana, Petunia hybrid and Antirrhinum majus by spatial restriction of ' $C$ ' class floral homeotic genes $[18,52]$. In congruent with these studies, our result shows a gradual difference in miR169 expression pattern among different sexes - highest in male ( $>5$ fold compared to female), intermediate in hermaphrodite ( $\sim 2$ fold compared to female) and lowest in female. The miRNAs expressed highest in the papaya female flowers (miR164, miR166, and miR394) are mainly involved in regulating the embryo patterning and floral meristem identity genes [53-55]. Although whether these differences are the cause or consequences of sexual dimorphism can not be concluded now, it is worth investigating these miRNAs for their roles in sex expression in papaya.

\section{Conclusions}

Using the large scale sRNA sequences from the male, female and hermaphrodite flowers of papaya, we have identified, 1) the sRNA hotspot on the papaya sex chromosome reminiscent of pericentromeric region, and 2) differentially expressed miRNAs in the flowers of different sex types. The identified pericentromeric regions of the sex chromosomes are located adjacent to the unfilled gap on the physical map, indicating that the centromere of these chromosomes lies in the gap. Relative position of the pericentromeric region on $\mathrm{X}$ and $\mathrm{Y}$ chromosomes revealed that the centromere of $\mathrm{X}$ and $\mathrm{Y}$ chromosomes are located 1.6 Mb apart from each other, indicating that the inversion on Y chromosome occurred at the pericentromeric region spanning the centromere. Our results provide valuable information for further characterization of papaya sex chromosomes.

By analyzing the miRNA expression in papaya flowers, we have identified 12 miRNAs differentially expressed among the three sex types. Majority of the miRNAs expressed higher in male flower targets the genes involved in auxin signaling pathway. We observed a higher expression of miR169 in male flowers, which has been implicated to repress ' $C$ ' class floral homeotic genes in other plant species. Further functional analysis of these miRNAs may reveal their role in papaya sex determination.

\section{Methods \\ Small RNA library construction and sequencing}

The small RNA libraries were constructed from male, female and hermaphrodite flowers of papaya. Mixture of flowers at different developmental stage, including young bud to fully mature flowers, was used for RNA extraction. Illumina sRNA library was prepared for each sex type as described previously [56]. In brief, total RNA was isolated using the Trizol reagent (Invitrogen USA). Low molecular weight RNA was enriched from the total RNA by precipitating with $0.4 \mathrm{M} \mathrm{NaCl}$ and polyethylene glycol (PEG). The enriched low molecular weight RNA was then separated on a denaturing $15 \%$ polyacrylamide gel. The band corresponding to the RNA standard of 18-30 nucleotides was then excised from the gel and eluted overnight in $0.4 \mathrm{M} \mathrm{NaCl}$ at $4^{\circ} \mathrm{C}$, and subsequently ligated with 5' and 3' Illumina small RNA adapters. The adapter-ligated library was converted into cDNA and amplified by using PCR and sequenced by Illumina Genome Analyzer II.

\section{Identification of miRNAs from sRNA dataset}

Computational analysis of sRNA reads obtained from the sRNA libraries was performed as reported previously $[57,58]$. Briefly, sRNA reads were extracted after trimming the adaptor sequence and then created an unique small RNA data set along with their read counts. The unique sRNAs were aligned to REPBASE version 14, (http://www.girinst.org), and other classes of known noncoding RNAs (rRNAs, tRNAs, snRNAs, snoRNAs, etc.) obtained from RFAM database (http://www.sanger. ac.uk/Software/Rfam/ftp.shtml). Following the removal of sRNAs corresponding to repetitive elements and known ncRNAs (rRNAs, tRNAs and other messenger RNAs), conserved miRNAs were identified by aligning the unique sRNA reads to the miRBase version 15 (http://microrna.sanger.ac.uk/). For identification of novel miRNAs, unique sRNAs with more than 10 genomic hits were removed from further analysis. The flanking regions of the remaining genome-matched sequences were extracted and the fold-back structures were predicted using the RNAfold program [59]. Next, we examined the resulting folding structures to choose those that had at least 18 base-pairs, one central loop, and folding energy not greater than $18 \mathrm{Kcal} / \mathrm{mol}$. The fold-back structures that meet the accepted criteria for miRNA annotation, $\leq 6$ mismatches, $\leq 2$ bulged or asymmetrically unpaired nucleotides, and $\leq 2$ continuous 
mismatches in the putative miRNA region, were selected using MIRCHECK program [60]. Subsequently, a program was developed in house to check the existence of miRNA* sequence of the selected mature miRNA, based on the criterion that there were 2nt overhang(s) at the 3' end(s) of either the miRNA or miRNA*.

\section{Mapping of sRNAs reads on to the sex chromosomes}

The sRNA reads were aligned to the papaya sex chromosome pseudomolecules by using the 'bowtie' short read aligner. The bowtie option was set to obtain only the reads that match $100 \%$ to the reference. The SAM output from bowtie was converted binary file (BAM) using Samtools. The BAM output was then used to generate the alignment map using the Bamview program. For the conservation analysis between the pericentromeric regions of the sex chromosomes, we manually extracted the sRNA hotspots from each chromosomes based on the alignment maps generated above. Subsequently, the reads mapped to the $Y$ chromosome sRNA hotspot on Y chromosome was realigned to the $\mathrm{X}$ chromosome hotspot and vice versa. The papaya sex specific small RNA sequence data used in this study can be obtained from NCBI's Gene Expression Ombibus (GEO) using accession number GSE54097.

\section{Additional file}

Additional file 1: Table S1. Summary of the sRNA reads mapped to the papaya sex chromosomes. Figure S1. Distribution of purine-rich and pyrimidine-rich sequences in the sRNA libraries. Figure S2. Frequency of different nucleotides en each position of 21 and 24 nt sequences. Figure S3. Precursor fold back structures of the newly identified miRNAs in papaya.

\section{Competing interests}

The authors declare that they have no competing interests.

\section{Authors' contributions}

RA, GJ, YZ, and QY conducted lab and field experiments, and bioinformatics analysis. RS and RM conceived the study, coordinated research activities. RA, $\mathrm{RS}$, and RM wrote the manuscript; all authors read and approved the final manuscript.

\section{Acknowledgements}

We would like to thank Dr. Stephen Downie, Marija Pushko, and Will Wadlington for editing and providing feedbacks on the manuscript. This work was supported by a grant from the National Science Foundation (NSF) Plant Genome Research Program to RM and QY (Award Nos. DBI 0922545). RS acknowledges the support from the Oklahoma Agricultural Experiment Station.

\section{Author details}

'Department of Plant Biology, University of Illinois at Urbana-Champaign, Urbana, IL 61801, USA. ²Department of Biochemistry and Molecular Biology, Oklahoma State University, Stillwater, OK 74078, USA. ${ }^{3}$ Faculty of Life Science and Technology, Kunming University of Science and Technology, Kunming, Yunnan 650500, China. ${ }^{4}$ Department of Plant Pathology \& Microbiology, Texas A\&M AgriLife Research Center, Texas A\&M University System, Dallas, TX75252, USA. 'FAFU and UIUC-SIB Joint Center for Genomics and Biotechnology, Fujian Agriculture and Forestry University, Fuzhou, Fujian 350002, China.
Received: 3 October 2013 Accepted: 30 December 2013

Published: 13 January 2014

\section{References}

1. Axtell MJ, Snyder JA, Bartel DP: Common functions for diverse small RNAs of land plants. Plant Cell 2007, 19(6):1750-1769.

2. Bartel B, Bartel DP: MicroRNAs: at the root of plant development? Plant Physiol 2003, 132(2):709-717.

3. Bartel DP: MicroRNAs: genomics, biogenesis, mechanism, and function. Cell 2004, 116:281-297.

4. Reinhart BJ, Weinstein EG, Rhoades MW, Bartel B, Bartel DP: MicroRNAs in plants. Genes Dev 2002, 16(13):1616-1626.

5. Rhoades MW, Reinhart BJ, Lim LP, Burge CB, Bartel B, Bartel DP: Prediction of plant microRNA targets. Cell 2002, 110:513-520.

6. Bernstein E, Allis CD: RNA meets chromatin. Genes Dev 2005 19(14):1635-1655.

7. Cantu D, Vanzetti LS, Sumner A, Dubcovsky M, Matvienko M, Distelfeld A, Michelmore RW, Dubcovsky J: Small RNAs, DNA methylation and transposable elements in wheat. BMC Genomics 2010, 11:408.

8. Axtell MJ: Classification and comparison of small RNAs from plants. Annu Rev Plant Biol 2013, 64:137-159.

9. Sunkar R, Chinnusamy V, Zhu J, Zhu JK: Small RNAs as big players in plant abiotic stress responses and nutrient deprivation. Trends Plant Sci 2007, 12(7):301-309.

10. Grant-Downton R, Hafidh S, Twell D, Dickinson HG: Small RNA pathways are present and functional in the angiosperm male gametophyte. Mol Plant 2009, 2(3):500-512.

11. Olmedo-Monfil V, Duran-Figueroa N, Arteaga-Vazquez M, Demesa-Arevalo E, Autran D, Grimanelli D, Slotkin RK, Martienssen RA, Vielle-Calzada J-P: Control of female gamete formation by a small RNA pathway in Arabidopsis. Nature 2010, 464(11):628-634.

12. Wu G, Park MY, Conway SR, Wang JW, Weigel D, Poethig RS: The sequential action of miR156 and miR172 regulates developmental timing in Arabidopsis. Cell 2009, 138(4):750-759.

13. Kim JJ, Lee JH, Kim W, Jung HS, Huijser P, Ahn JH: The microRNA156SQUAMOSA PROMOTER BINDING PROTEIN-LIKE3 module regulates ambient temperature-responsive flowering via FLOWERING LOCUS T in Arabidopsis. Plant Physiol 2012, 159(1):461-478.

14. Nodine MD, Bartel DP: MicroRNAs prevent precocious gene expression and enable pattern formation during plant embryogenesis. Genes Dev 2010, 24(23):2678-2692.

15. Juarez MT, Kui JS, Thomas J, Heller BA, Timmermans MCP: microRNAmediated repression of rolled leaf1 specifies maize leaf polarity. Nature 2004, 428:84-88.

16. Achard P, Herr A, Baulcombe DC, Harberd NP: Modulation of floral development by a gibberellin-regulated microRNA. Development 2004 131(14):3357-3365.

17. Aukerman MJ, Sakai H: Regulation of flowering time and floral organ identity by microRNA and its Apatala2-like target gene. Plant Cell 2003, 15(11):2730-2741.

18. Cartolano M, Castillo R, Efremova N, Kuckenberg M, Zethof J, Gerats T, Schwarz-Sommer Z, Vandenbussche M: A conserved microRNA module exerts homeotic control over Petunia hybrida and Antirrhinum majus floral organ identity. Nat Genet 2007, 39(7):901-905.

19. Chen $X: A$ microRNA as a translational repressor of APETALA2 in Arabidopsis flower development. Science 2004, 303(5666):2022-2025.

20. Yamaguchi A, Wu MF, Yang L, Wu G, Poethig RS, Wagner D: The microRNAregulated SBP-Box transcription factor SPL3 is a direct upstream activator of LEAFY, FRUITFULL, and APETALA1. Dev Cell 2009, 17(2):268-278.

21. Zhao $L$, Kim $Y$, Dinh $\Pi$, Chen $X$ : miR172 regulates stem cell fate and defines the inner boundary of APETALA3 and PISTILLATA expression domain in Arabidopsis floral meristems. Plant J 2007, 51(5):840-849.

22. Banks JA: MicroRNA, sex determination and floral meristem determinacy in maize. Genome Biol 2008, 9(1):204.

23. Chuck G, Meeley R, Irish E, Sakai H, Hake S: The maize tasselseed4 microRNA controls sex determination and meristem cell fate by targeting Tasselseed6/indeterminate spikelet1. Nat Genet 2007, 39(12):1517-1521

24. She X, Xu X, Fedotov A, Kelly WG, Maine EM: Regulation of heterochromatin assembly on unpaired chromosomes during 
Caenorhabditis elegans meiosis by components of a small RNA-mediated pathway. PLoS Genet 2009, 5(8):e1000624.

25. Carvalho FA, Renner SS: A dated phylogeny of the papaya family (Caricaceae) reveals the crop's closest relatives and the family's biogeographic history. Mol Phylogen Evol 2012, 65(1):46-53.

26. Storey WB: Genetics of the papaya. J Hered 1953, 44:70-78

27. Ming $R$, Yu Q, Moore PH: Sex determination in papaya. Semin Cell Dev Biol 2007, 18(3):401-408.

28. Yu Q, Navajas-Pérez R, Tong E, Robertson J, Moore PH, Paterson AH, Ming R: Recent origin of dioecious and gynodioecious $Y$ chromosomes in papaya. Tropical Plant Biology 2008, 1(1):49-57.

29. Zhang W, Wang X, Yu Q, Ming R, Jiang J: DNA methylation and heterochromatinization in the male-specific region of the primitive $Y$ chromosome of papaya. Genome Res 2008, 18(12):1938-1943.

30. Wang J, Na J-K, Yu Q, Gschwend AR, Han J, Zeng F, Aryal R, VanBuren R, Murray JE, Zhang $W$, et al: Sequencing papaya $X$ and $Y h$ chromosomes revealed molecular basis of incipient sex chromosome evolution. Proc Natl Acad Sci USA 2012, 109(34):13710-13715.

31. Gschwend AR, Yu Q, Tong EJ, Zeng F, Han J, VanBuren R, Aryal R, Charlesworth D, Moore PH, Paterson AH, et al: Rapid divergence and expansion of the $\mathrm{X}$ chromosome in papaya. Proc Natl Acad Sci USA 2012, 109(34):13716-13721.

32. Aryal R, Yang X, Yu Q, Sunkar R, Li L, Ming R: Asymmetric purinepyrimidine distribution in cellular small RNA population of papaya. BMC Genomics 2012, 13:682.

33. Aryal $R$, Ming $R$ : Sex determination in flowering plants: papaya as a model system. Plant Sci 2013. http://dx.doi.org/10.1016/j. plantsci.2013.10.018.

34. Meyers BC, Axtell MJ, Bartel B, Bartel DP, Baulcombe D, Bowman JL, Cao X, Carrington JC, Chen X, Green PJ, et al: Criteria for annotation of plant MicroRNAs. Plant Cell 2008, 20(12):3186-3190.

35. Porter BW, Aizawa KS, Zhu YJ, Christopher DA: Differentially expressed and new non-protein-coding genes from a Carica papaya root transcriptome survey. Plant Sci 2008, 174(1):38-50.

36. Chen ZH, Bao ML, Sun YZ, Yang YJ, Xu XH, Wang JH, Han N, Bian HW, Zhu MY: Regulation of auxin response by miR393-targeted transport inhibitor response protein 1 is involved in normal development in Arabidopsis. Plant Mol Biol 2011, 77(6):619-629.

37. Mallory AC, Bartel DP, Bartel B: MicroRNA-directed regulation of Arabidopsis AUXIN RESPONSE FACTOR17 is essential for proper development and modulates expression of early auxin response genes. Plant Cell 2005, 17(5):1360-1375.

38. Liu Q, Zhang YC, Wang CY, Luo YC, Huang QJ, Chen SY, Zhou H, Qu LH, Chen YQ: Expression analysis of phytohormone-regulated microRNAs in rice, implying their regulation roles in plant hormone signaling. FEBS Lett 2009, 583(4):723-728.

39. Na JK, Wang J, Murray JE, Gschwend AR, Zhang W, Yu Q, Navajas-Perez R, Feltus FA, Chen C, Kubat Z, et al: Construction of physical maps for the sex-specific regions of papaya sex chromosomes. BMC Genomics 2012, 13(1):176.

40. Reinhart BJ, Bartel DP: Small RNAs correspond to centromere heterochromatic repeats. Science 2002, 297(5588):1831.

41. Yu Q, Hou S, Hobza R, Feltus FA, Wang X, Jin W, Skelton RL, Blas A, Lemke C, Saw JH, et al: Chromosomal location and gene paucity of the male specific region on papaya Y chromosome. Mol Genet Genomics 2007, 278(2):177-185.

42. Ma H, Moore PH, Liu Z, Kim MS, Yu Q, Fitch MMM, Sekioka T, Paterson AH, Ming R: High-density linkage mapping revealed suppression of recombination at the sex determination locus in papaya. Genetics 2004, 166:419-436.

43. Liu Z, Moore PH, Ma H, Ackerman CM, Ragiba M, Yu Q, Pearl HM, Kim MS, Charlton JW, Stiles Jl, et al: A primitive $Y$ chromosome in papaya marks incipient sex chromosome evolution. Nature 2004, 427:348-352.

44. The International Brachypodium Initiative: Genome sequencing and analysis of the model grass Brachypodium distachyon. Nature 2010, 463(7282):763-768.

45. Lu C, Tej SS, Luo S, Haudenschild CD, Meyers BC, Green PJ: Elucidation of the small RNA component of the transcriptome. Science 2005, 309(5740):1567-1569.

46. Lu C, Kulkarni K, Souret FF, MuthuValliappan R, Tej SS, Poethig RS, Henderson IR, Jacobsen SE, Wang W, Green PJ, et al: MicroRNAs and other small RNAs enriched in the Arabidopsis RNA-dependent RNA polymerase-2 mutant. Genome Res 2006, 16(10):1276-1288.

47. Hayden KE, Willard HF: Composition and organization of active centromere sequences in complex genomes. BMC Genomics 2012, 13:324.

48. Nagaki K, Cheng Z, Ouyang S, Talbert PB, Kim M, Jones KM, Henikoff S, Buell $C R$, Jiang J: Sequencing of a rice centromere uncovers active genes. Nat Genet 2004, 36(2):138-145.

49. Kumar LSS, Abraham A, Srinivasan VK: The cytology of Carica papaya Linn. Indian J Agr SCi 1945, 15:242-253.

50. Liu Q, Chen YQ: Insights into the mechanism of plant development: interactions of miRNAs pathway with phytohormone response. Biochem Biophys Res Commun 2009, 384(1):1-5.

51. Larsson E, Franks RG, Sundberg E: Auxin and the Arabidopsis thaliana gynoecium. J Exp Bot 2013, 64(9):2619-2627.

52. Geuten K, Irish V: Hidden variability of floral homeotic $B$ genes in Solanaceae provides a molecular basis for the evolution of novel functions. Plant Cell 2010, 22(8):2562-2578.

53. Mallory AC, Dugas DV, Bartel DP, Bartel B: MicroRNA regulation of NACdomain targets is required for proper formation and separation of adjacent embryonic, vegetative, and floral organs. Curr Biol 2004, 14(12):1035-1046.

54. Jung JH, Park CM: MIR166/165 genes exhibit dynamic expression patterns in regulating shoot apical meristem and floral development in Arabidopsis. Planta 2007, 225(6):1327-1338.

55. Knauer S, Holt AL, Rubio-Somoza I, Tucker EJ, Hinze A, Pisch M, Javelle M, Timmermans MC, Tucker MR, Laux T: A protodermal miR394 signal defines a region of stem cell competence in the Arabidopsis shoot meristem. Dev Cell 2013, 24(2):125-132.

56. Sunkar R, Zhou X, Zheng Y, Zhang W, Zhu JK: Identification of novel and candidate miRNAs in rice by high throughput sequencing. BMC Plant Biol 2008, 8:25.

57. Jagadeeswaran G, Nimmakayala P, Zheng Y, Gowdu K, Reddy UK, Sunkar R: Characterization of the small RNA component of leaves and fruits from four different cucurbit species. BMC Genomics 2012, 13:329.

58. Jagadeeswaran G, Zheng Y, Sumathipala N, Jiang H, Arrese EL, Soulages JL, Zhang W, Sunkar R: Deep sequencing of small RNA libraries reveals dynamic regulation of conserved and novel microRNAs and microRNAstars during silkworm development. BMC Genomics 2010, 11:52.

59. Hofacker IL: Vienna RNA secondary structure server. Nucleic Acids Res 2003 31(13):3429-3431.

60. Jones-Rhoades MW, Bartel DP: Computational identification of plant microRNAs and their targets, including a stress-induced miRNA Mol Cell 2004, 14(6):787-799.

doi:10.1186/1471-2164-15-20

Cite this article as: Aryal et al: Sex specific expression and distribution of small RNAs in papaya. BMC Genomics 2014 15:20.

\section{Submit your next manuscript to BioMed Central and take full advantage of:}

- Convenient online submission

- Thorough peer review

- No space constraints or color figure charges

- Immediate publication on acceptance

- Inclusion in PubMed, CAS, Scopus and Google Scholar

- Research which is freely available for redistribution 